\title{
MODELO DE GESTIÓN DE LAS TIC PARA LAS PYMES DE RIOBAMBA (ECUADOR)
}

\section{ICT MANAGEMENT MODEL FOR RIOBAMBA SMES (ECUADOR)}

Maria Sluzarczyk Antosz ${ }^{1}$

1. Ingeniera Civil (Politécnica de Cracovia - Polonia). Máster en Informática Aplicad (Escuela Superior Politécnica de Chimborazo- Ecuador). Máster en Gerencia y Liderazgo Educacional (UTPL - Ecuador). Docente de la Facultad de Administración de Empresas en la Escuela Superior Politécnica de Chimborazo (ESPOCH), Riobamba - Ecuador. Directora de la Unidad de Educación a Distancia ESPOCH.

\section{Citación sugerida:}

Sluzarczyk Antosz, M. (2017). Modelo de gestión de las TIC para las pymes de Riobamba (Ecuador). 3C TIC: Cuadernos de desarrollo aplicados a las TIC, 6(4), 1-18. DOI: <http://dx.doi.org/10.17993/3ctic.2017.58.1-18/>. 


\section{RESUMEN}

La mayoría de las economías actuales tanto a nivel mundial, como latinoamericano y ecuatoriano se componen en gran parte de pequeñas y medianas empresas (PyMEs), que realizan una importante contribución a la industria y al empleo. Sin embargo existe un factor esencial para la supervivencia, la rentabilidad y crecimiento de las PyMEs, que es la innovación y adecuada utilización de las Tecnologías de la Información y la Comunicación TIC.

El presente trabajo tiene como objetivo principal presentar un modelo de gestión de las TIC para las PyMEs de Riobamba - Ecuador, que toma en cuenta las características de dichas organizaciones empresariales. En investigaciones previas se estudió la literatura especializada en el área de las TIC, su impacto en la gestión empresarial, se analizaron los modelos de gestión de las TIC existentes y se realizó el diagnóstico de utilización de las TIC en las PyMEs de Riobamba. En base a dicha información se estructura un modelo de gestión de las TIC para las PyMEs de Riobamba, presentando aquí sus principios, enfoques, componentes, variables y su representación gráfica. El modelo es validado por los expertos en el área de TIC y PyMEs.

\section{ABSTRACT}

Most of today's global, Latin American and Ecuadorian economies are largely made up of small and medium-sized enterprises (SMEs), which make a major contribution to industry and employment. However, there is an essential factor for SMEs survival, profitability and growth, which is innovation and adequate use of Information and Communication Technologies - ICT.

The main objective of this paper is to present a ICT management model for SMEs in Riobamba - Ecuador, which takes into account the characteristics of these business organizations. In previous research, specialized literature was studied in the area of ICT, its impact on business management, the analysis of existing ICT management models and the diagnosis of the use of ICT in Riobamba SMEs. Based on all this information, a ICT management model for Riobamba SMEs is structured, presenting here its principles, approaches, components, variables and its graphic representation. The model is validated by experts in the area of ICT and SMEs.

\section{PALABRAS CLAVE}

Modelo de gestión, TIC, PyMEs, método Delphi, método ANOCHI.

\section{KEYWORDS}

Management model, ICTs, SMEs, Delphi method, ANOCHI method. 


\section{INTRODUCCIÓN}

La investigación de campo realizada (Slusarczyk, 2015a) muestra que la mayoría de las PyMEs de Riobamba han implementado herramientas básicas de TIC y que su función operativa es muy importante. Sin embargo, es necesario crear un espacio donde estas herramientas tengan un rol estratégico y sean aplicadas en forma efectiva.

La investigación teórica y el análisis respecto a las posibilidades de utilización de las TIC en las empresas en el mundo actual globalizado, la discusión con los especialistas y directivos vinculados a las TIC y las PyMEs en Riobamba, el análisis comparativo de diferentes modelos de gestión de las TIC en las empresas (Slusarczyk, 2015b), fundamentan la necesidad de diseñar un modelo de gestión de las TIC.

Este modelo permitirá efectuar los cambios en la manera como las PyMEs utilizan, administran y alinean estas herramientas informáticas con los objetivos del negocio.

Además existe la necesidad de elevar el desarrollo productivo y socioeconómico de todo tipo de empresas, incluidas las PyMEs y de ayudar a que su gestión sea más eficiente. De hecho, la aplicación de un modelo de gestión de las TIC garantiza una mejor gestión, control y toma de decisiones en estas organizaciones, lo que está en concordancia con las políticas del gobierno de Ecuador que pretende reforzar la matriz productiva.

El objetivo general del modelo que se propone es establecer un marco de referencia para la gestión de las TIC en las PyMEs, tomando en cuenta su entorno político, social, económico, legal y tecnológico, como otros elementos con el propósito de alinear la estrategia de las TIC con los objetivos de la empresa.

\section{ENFOQUES Y PRINCIPIOS DEL MODELO}

Para que el modelo tenga una buena base teórica, debe sustentarse en: objetivos, premisas, enfoques y principios (Torres, 2015).En la creación de este modelo se utilizan los siguientes enfoques:

- Enfoque de contingencia (situacional): considera que no existe una forma única para alcanzar los objetivos y las metas de la empresa. Las mejores técnicas administrativas, para alcanzar las metas de la organización pueden variar de acuerdo con las situaciones o circunstancias y en gran medida dependen de la tecnología y del ambiente externo condiciones legales, políticas, económicas, culturales (Sánchez, 2011).

- Enfoque de sistema: señala que la integración de las TIC con el negocio es más que una simple suma de sus partes, es una cualidad surgida de la interacción de sus partes, llamada alineación estratégica entre las TIC y la organización. Además las empresas no existen en el vacío, sino que dependen de condiciones externas y forman parte de sistemas más grandes, como la industria a la que pertenecen, el sistema económico y la sociedad en la cual están insertas (Kast, 1992).

- Enfoque de liderazgo de la dirección. Reconoce la importancia del liderazgo en la empresa. El modelo contiene un espacio que son las juntas de trabajo donde se puede 


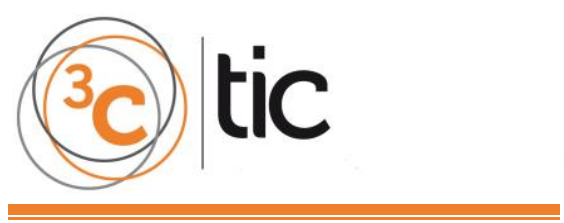

poner en funcionamiento buenas prácticas de liderazgo como: motivación de los empleados para que se comprometan con los objetivos de la empresa, trato justo, firmeza, empatía, disciplina, capacidad para envisionar un futuro mejor, etc. El éxito de una PyME depende en gran medida del acertado liderazgo en todos los niveles de la organización (Barroso, 2005; Malaret, 2003).

- Enfoque tecnológico: las tecnologías son la base del funcionamiento de las TIC y su incidencia en el desarrollo, competitividad y productividad de las PyMEs es reconocida mundialmente. Por lo tanto es importante crear una cultura e infraestructura que permita la adopción de las nuevas tecnologías. La gestión de las tecnologías emergentes exige el empleo de enfoques diferentes en varios aspectos como estrategias, aplicación de conocimientos, habilidades y toma de decisiones (González, 2012; Ochoa, 2007).

- Enfoque estratégico:es saber qué tan cerca están relacionadas las estrategias de sistemas de información y comunicación con el planteamiento y cumplimiento de las metas globales de la organización. Esto significa entender cuál es el papel que juegan las TIC en las estrategias del negocio y crear estrategias adecuadas según el impacto de las condiciones externas cambiantes (Gimbert, 2007).

- Enfoque proactivo: en organizaciones sometidas a cambios rápidos, como ocurre actualmente, se debe ser proactivo y anticiparse a los acontecimientos, basándose en el análisis de la información con objeto de reducir la incertidumbre. La administración proactiva, que es contraria a la reactiva, busca adelantarse a los acontecimientos problemáticos, a través de herramientas como el análisis causal, la toma de decisiones innovadora y el análisis de la planificación, con el fin de plantear soluciones en función de las necesidades (Universidad de Champagnat, 2002).

El modelo propuesto se sustenta en los siguientes principios:

- Mejora continua: significa que este modelo podrá ser mejorado, si las futuras necesidades o experiencias así lo requieren, sobre todo, después de aplicarlo dentro de una organización y en constante cambio. También, el modelo dentro de sí contiene un ciclo de mejora continua, el cual permite a la empresa una mejora integral.

- Comunicación y cooperación entre los ejecutivos y empleados: este principio ayuda a lograr la alineación estratégica de las TIC con los objetivos del negocio. Si se coordinan las actividades y se toman las decisiones en base a una información confiable y con la participación de personas implicadas, el desempeño de la empresa será más efectivo. Por lo tanto la PyME que desea aplicar en forma efectiva el modelo presentado adelante, debe hacer cambios en su forma de gestionar los recursos humanos incorporando en su liderazgo elementos como: conocimiento, dominio de los procesos productivos, reconocimiento e involucramiento.

Por la importancia y responsabilidades que tienen los jefes de las TIC se debe poner mucha atención en el momento de su contratación. Generalmente el jefe de TIC no forma parte de la Junta Directiva, lo que genera mayores riesgos en cuanto al alineamiento estratégico que se espera que tengan las TIC. La razón para esta situación es el rol de soporte que históricamente han tenido las TIC en las empresas a lo largo del tiempo, un soporte de tipo netamente operativo, orientado a la automatización de los procesos (Peirano y Suárez, 2006). 
Como se puede apreciar en la figura 1, por sus múltiples responsabilidades, el jefe de las TIC debe ser miembro del comité de dirección para ampliar el impacto empresarial y contribuir a la reducción de los costos, lo que se toma en cuenta en el modelo propuesto.

Figura 1. El rol del jefe de las TIC en la empresa.

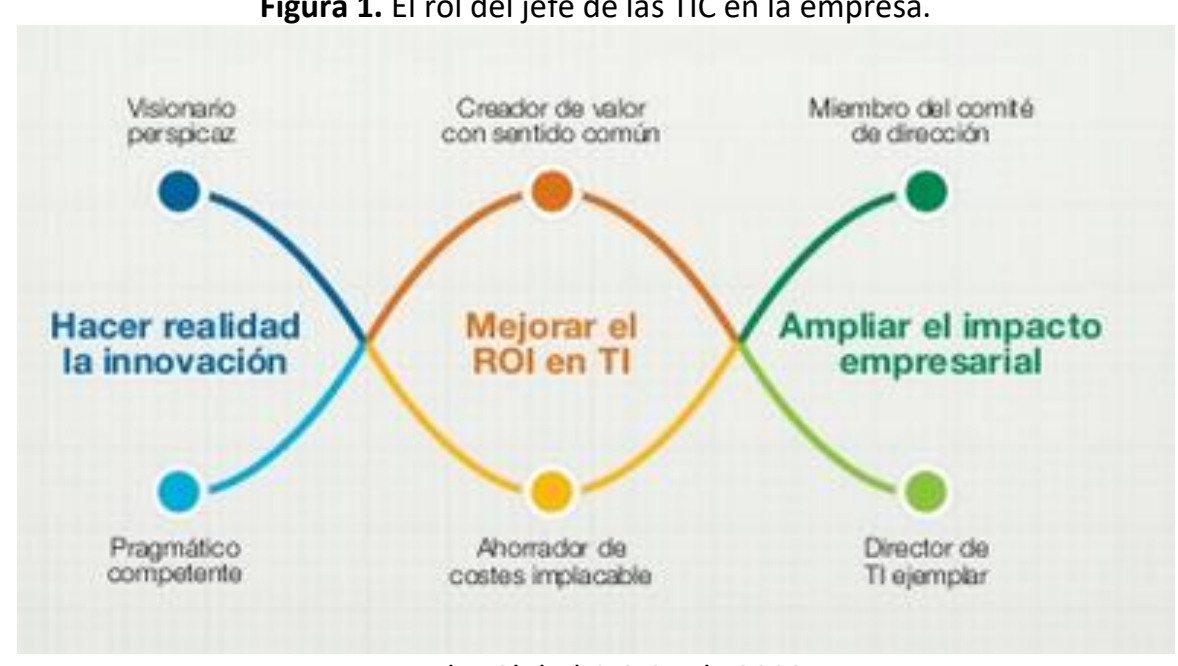

Fuente: The Global CIO Study 2009 IBM.

Según IT Governance Institute (2007), solo un poco más de la mitad de los jefes de TIC forman parte del comité de dirección de la empresa y el $42 \%$ de ellos reportan a la gerencia financiera de la empresa y no al comité.

- Capacidad de aprender, desaprender y adaptarse: actualmente con numerosos cambios a nivel tecnológico, con tantas nuevas estrategias empresariales, con inestabilidad en todos los aspectos relacionados con la vida personal y profesional, es necesario que el capital humano de la empresa sea muy flexible en lo que se refiere a aprender, desaprender y adaptarse. Esto es muy importante en el caso del personal relacionado con las nuevas tecnologías para que pueda proponer e instalar en la empresa las mejores y más adecuadas soluciones informáticas para el perfil y estrategias de las PyMEs.

- Creatividad e innovación: consiste en crear cosas nuevas, hallar soluciones originales, encontrar procedimientos o elementos para desarrollar labores de manera distinta a la tradicional. La creatividad puede ser un recurso que favorezca la productividad de la empresa y es un motor de innovación empresarial. La aplicación de este modelo abre espacio a una comunicación más abierta, conocimiento de las necesidades y estrategias del negocio, mejores prácticas en el liderazgo, lo cualpuede influir en forma positiva en la creatividad e innovación.

- Austeridad y ahorro: la coyuntura económica de la crisis general y los recortes presupuestarios exigen a las PyMEs establecer reglas para el ahorro y gasto eficiente. Se deben optimizar los recursos, eliminar trámites y gastos innecesarios, reducir los costos de las operaciones y administración, y establecer los mecanismos de control. Para lograr estos objetivos un rol importante lo juegan las TIC. Este principio se cumple mediante la organización centralizada de las TIC, aplicación del círculo de mejora continua y a través de las juntas de trabajo donde se gestionan los recursos. 


\section{COMPONENTES DEL MODELO Y SUS INTERRELACIONES}

Los análisis desarrollados crean las bases teóricas y empíricas para la propuesta del modelo mostrado en la figura 2, concebido con un carácter integral. El modelo se basa en los elementos comunes encontrados en los diferentes factores que inciden en la gestión de las TIC en las PyMEs.

\subsection{FACTORES EXTERNOS}

Se toma en cuenta el entorno y la influencia de los factores externos, entre los cuales es preciso considerar las políticas de estado ecuatoriano para la informatización a través del Ministerio de Telecomunicaciones y de la Sociedad de la Información (2014). La creación de este ministerio responde a la necesidad de coordinar acciones de apoyo y asesoría para garantizar el acceso igualitario a los servicios relacionados con el área de telecomunicación, para de esta forma asegurar el avance hacia la Sociedad de la Información y así el buen vivir de la población ecuatoriana.

Para garantizar el cumplimiento de requerimientos externos se debe identificar leyes nacionales y locales, regulaciones, y otros requerimientos externos legales y regulatorios que se deben cumplir para incorporarlos en las políticas de la organización (IT Governance Institute, 2007).

Otros factores externos son: lineamientos de la Cámara de Pequeña Industria de Chimborazo y la Cámara de Comercio de Riobamba para el impulso de la informatización, políticas sectoriales para el impulso de las nuevas tecnologías en las PyMEs, ambiente tecnológico externo (infraestructura de telecomunicaciones, disponibilidad de las soluciones requeridas), políticas empresariales, entorno social y económico, el marco legal que existe en el país referente a: facturas electrónicas, correo electrónico y firmas electrónicas.

Todo esto se considera para analizar el entorno social y económico que rodea la gestión de las TIC, como también el marco ético y legislativo. Los elementos mencionados del marco externo impactan directamente otras variables concebidas en el modelo como: estrategia e infraestructura de la PyME, estrategia e infraestructura de las TIC y el proceso de gestión de las TIC.

\subsection{ESTRATEGIAS DE LA EMPRESA Y DE LAS TIC}

Como se puede apreciar en la figura 2, entre la empresa y el departamento de las TIC existe mutua interdependencia e influencia. Considerando que este análisis se hace bajo un enfoque estratégico y tecnológico, es necesario que la estrategia del negocio siente las bases para la estrategia de las TIC, la cual a su vez, debe estar habilitada por la arquitectura de las TIC. De un lado, la PyME conduce, empodera e invierte en TIC y a través de ellas se impulsa la innovación, el crecimiento y la eficiencia en la empresa. En este modelo las estrategias ocupan un lugar fundamental tanto de la empresa como de las TIC. La estrategia del negocio se considera como punto de partida y ella define los procesos e infraestructura organizacional. La estructura organizacional de la empresa juega un papel importante para lograr el alineamiento estratégico, pues define las posiciones gerenciales que tendrá la PyME, 
los roles de cada uno de los jefes y de los empleados, como también la forma que estos interaccionan con la Junta Directiva.

De igual manera la estrategia de TIC que apoya la estrategia de la empresa, especifica los procesos e infraestructura de las TIC. Todos estos cuatro ámbitos estratégicos están interrelacionados entre sí.

Tanto por el tamaño de las PyMEs, como por el objetivo de cumplir con el principio de austeridad y ahorro, se considera una organización centralizada de las TIC a nivel corporativo y una organización interna de las TIC según las áreas funcionales de la empresa (Winkler, 2014). Existen diferentes herramientas TIC, que pueden ser aplicadas en diferentes ámbitos de la empresa con el objetivo de mejorar el desempeño empresarial: innovación, gestión financiera, promoción de la empresa, gestión empresarial, gestión de recursos humanos, capacitación, internacionalización, etc.

\subsection{JUNTAS DE TRABAJO}

En base al enfoque de liderazgo de la dirección y al principio de comunicación y cooperación entre ejecutivos y empleados, las juntas de trabajo constituyen un elemento central de este Modelo de Gestión de las TIC.

En estas juntas participan los ejecutivos de la empresa, tanto del área de TIC como de otras áreas, como también los empleados que desean aportar con sus ideas al desarrollo de la empresa. Estas juntas constituyen el espacio de: comunicación, liderazgo, cooperación, creatividad y toma de decisiones. Es importante que se tenga bien presente el tipo de negocio en el cual se compite y la posición de la empresa dentro de la industria.

Para que estas juntas cumplan con su propósito, cada reunión debe ser preparada con anticipación y bien dirigida. Se sugiere realización de tres tipos de reuniones: anual, mensual y quincenal. En la reunión anual el equipo directivo (jefes de áreas o personas que realizan funciones claves en la empresa) establece las metas financieras y otros objetivos de la PyME para los siguientes doce meses. Las metas anuales se dividen en metas mensuales y éstas son tratadas en las reuniones mensuales. En estas reuniones cada miembro debería poder identificar algunas metas específicas que se deben alcanzar en ese mes. La reunión quincenal es para dar seguimiento a las metas del mes y en caso que sea necesario replantear metas, desarrollar diferentes estrategias, etc.

En estas reuniones se realiza el diagnóstico de la situación de la empresa, se identifican oportunidades, se definen las prioridades, estrategias y los procesos de planificación, organización, dirección y control. Además los aspectos relacionados con las TIC tienen gran relevancia en la agenda de las reuniones; se analizan en forma permanente, tanto los aspectos operativos como estratégicos de las TIC.

También se sigue el progreso de los proyectos tecnológicos y se puntualizan las mejoras que deben darse en el departamento de las TIC para que en forma efectiva pueda apoyar los objetivos del negocio. Esto garantizaría que el departamento de las TIC esté al tanto de los planes, de los objetivos del negocio y pueda proponer las estrategias y acciones que se pueden realizar para apoyar dichos objetivos. 
En muchos aspectos, el alineamiento y efectiva gestión de las TIC en las empresas están determinados por la efectividad de las relaciones interpersonales entre los jefes de TIC y los jefes de otras áreas del negocio. Según Luftman (2000), los jefes de TIC, para ser eficaces, necesitan entre otras cosas conocer los planes tácticos y estratégicos de la dirección. Además, deberán estar presentes cuando se discutan las estrategias a nivel corporativo, juegan un papel dominante en las inversiones en TIC, para que éstas viabilicen el crecimiento de las organizaciones.

Figura 2. Representación gráfica del Modelo de Gestión de las TIC.

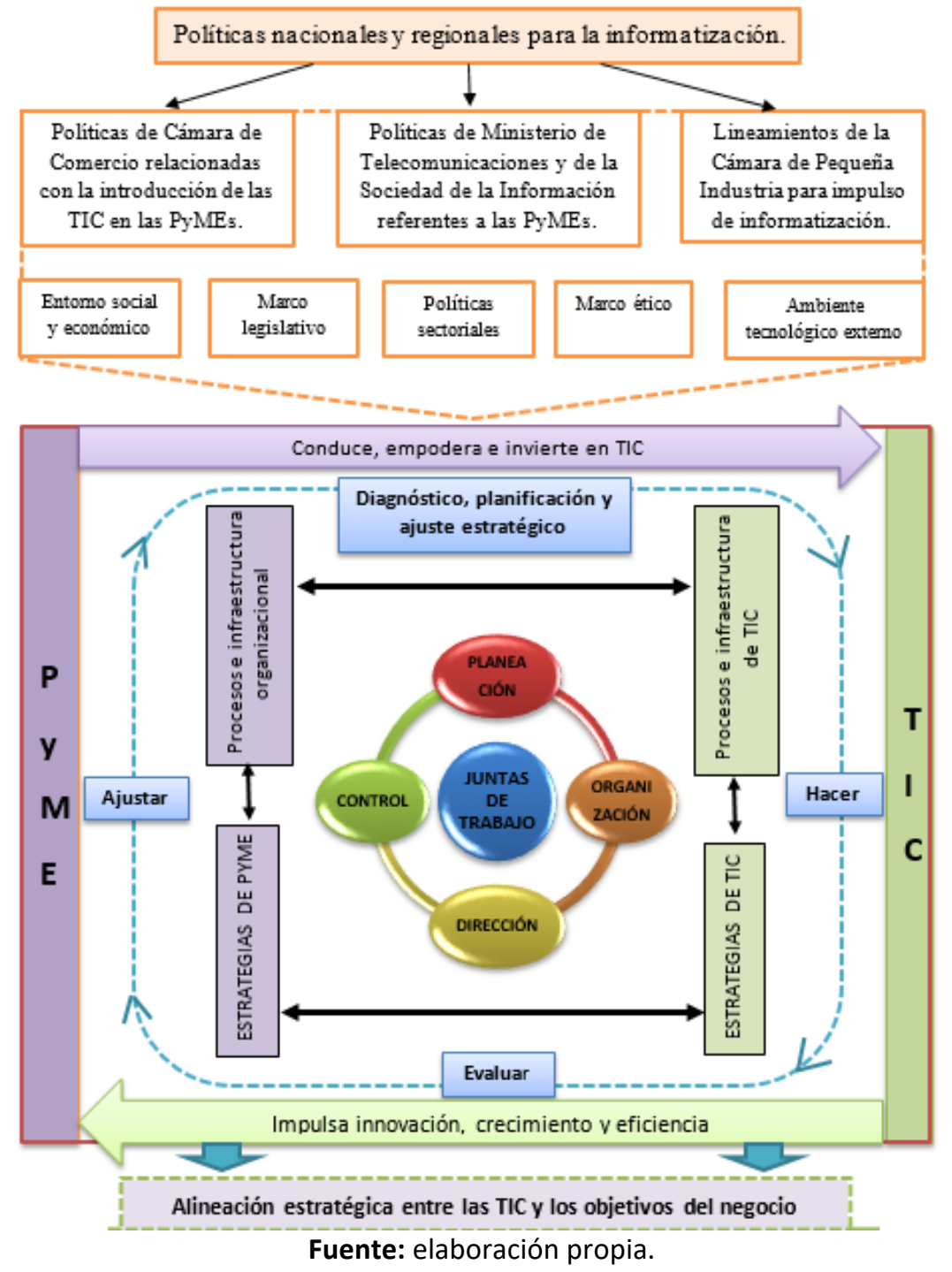

Según IT Governance Institute (2007) se debe facilitarseel entendimiento del consejo directivo y de los ejecutivos sobre temas estratégicos de TIC tales como: el rol de las TIC, características propias y capacidades de la tecnología. Asimismo, habría que garantizar la existencia de un entendimiento compartido entre el negocio y la función de TIC sobre la contribución potencial de las TIC a la estrategia del negocio, fomentar la co-responsabilidad entre las PyME y las TIC en la toma de decisiones estratégicas y en la obtención de los beneficios provenientes de las inversiones en las TIC. 
El Jefe de las TIC debe realizar informes para el consejo directivo sobre las estrategias, el desempeño y los riesgos de TIC. De igual manera el consejo directivo debe realizar informes sobre las TIC a los interesados.

\subsection{RIESGOS}

Se debe tomar en cuenta que invertir en materia de TIC, equivale a ingresar mucho dinero en equipo tecnológico (Ramón, 2014). Es de vital importancia que el software tenga fiabilidad, seguridad, facilidad de uso, eficiencia y eficacia para cada tipo de organización. Es por eso que las inversiones se deben realizar con especial cuidado. Los gastos en las TIC incluyen los componentes físicos (computadoras, dispositivos de almacenamiento, impresoras y otros periféricos); los programas de computación (sistemas operativos, herramientas de programación), servicios TIC y telecomunicaciones, siendo estos últimos, los que suponen mayores gastos.

Se requiere que los directivos y especialistas tengan conciencia de los riesgos en que se incurre al comprar tecnología de la información y la comunicación sin gestionarla y organizarla adecuadamente y que se garantice su seguridad. Para ello se debe: administrar la identidad de los usuarios, prevenir, detectar y corregir el software malicioso, garantizar que la información conficencial esté prohibida a aquéllos que no tienen acceso a ella, que las transacciones e intercambio de información automatizados sean confiables, mantener la integridad de la información, proteger y mantener el registro de todos los activos de TIC.

Kearney (2005) expresa que si los miembros del consejo directivo toman decisiones claves en el área de tecnología, los servicios valiosos de las TIC son implementados con mayor rapidez y las empresas superan a sus competidores en el crecimiento de los ingresos.

\subsection{MEJORA CONTINUA}

El modelo propuesto incluye el círculo de mejora continua de Deming (Deming, 2008; García 2016), el cual se compone de cuatro etapas cíclicas que se refieren a: planificar, hacer, evaluar y ajustar.

La planificación comprende básicamente establecer las actividades del proceso necesarias para obtener el resultado esperado. Con tal propósito se pueden utilizar algunas herramientas como:

- Tormenta de ideas con la participación de todos los interesados.

- Diagrama de Gantt para la planificación y seguimiento de las actividades.

- Análisis Modal de Fallos y Efectos (AMFE), que es un estudio de fallos potenciales y de sus consecuencias.

Hacer - significa realizar los cambios para implantar la mejora propuesta.

Evaluar (verificar) hace referencia al monitoreo de la implementación. Los datos de control son recopilados y analizados, comparándolos con los requisitos especificados inicialmente y documentando las conclusiones. Algunos ejemplos de herramientas de evaluación son:

- Diagrama de Pareto. Es una representación gráfica en forma de curva (80\%-20\%), que sirve para organizar datos y centrar los esfuerzos en lo más importante (pocos vitales). 
- Diagrama de correlación. Herramienta utilizada para la interpretación de datos utilizando el plano cartesiano. A través del mismo se puede examinar si existe relación entre dos variables y determinar si ésta es positiva o negativa.

- Diagrama de Ishikawa. Estudio para localizar las causas de los problemas.

La etapa de Ajustar parte de los resultados conseguidos en la fase anterior. Se procede a recopilar lo aprendido y a ponerlo en marcha, se formulan recomendaciones y observaciones que suelen servir para volver al paso inicial de Planificar.

Una vez acabada la etapa final se debe volver a la primera y repetir el ciclo de nuevo, las actividades son reevaluadas periódicamente para incorporar nuevas mejoras. La implementación de este ciclo permite lograr un mejoramiento de la calidad y competitividad.

Como resultado de la aplicación de este Modelo de Gestión de las TIC se espera lograr el alineamiento estratégico entre las TIC y los objetivos de la PyME, tomando en cuenta que esto no es un proyecto que se inicia y termina, más bien es un proceso y esfuerzo continuo que requiere saber tomar decisiones, riesgos y tener la capacidad de gestión y liderazgo (Constante y Quintana, 2014). Este alineamiento dependerá primordialmente del sector al cual pertenece la PyME y del grado de importancia que las TIC tienen en las operaciones y su estrategia.

El modelo propuesto presenta un enfoque sistémico y sinérgico ya que integra (triangula) en la organización: liderazgo y estrategia, las tecnologías y la mejora continua (ver figura 3).

Figura 3. Integración de estrategia, las tecnologías y la mejora continua en el Modelo de Gestión de las TIC.

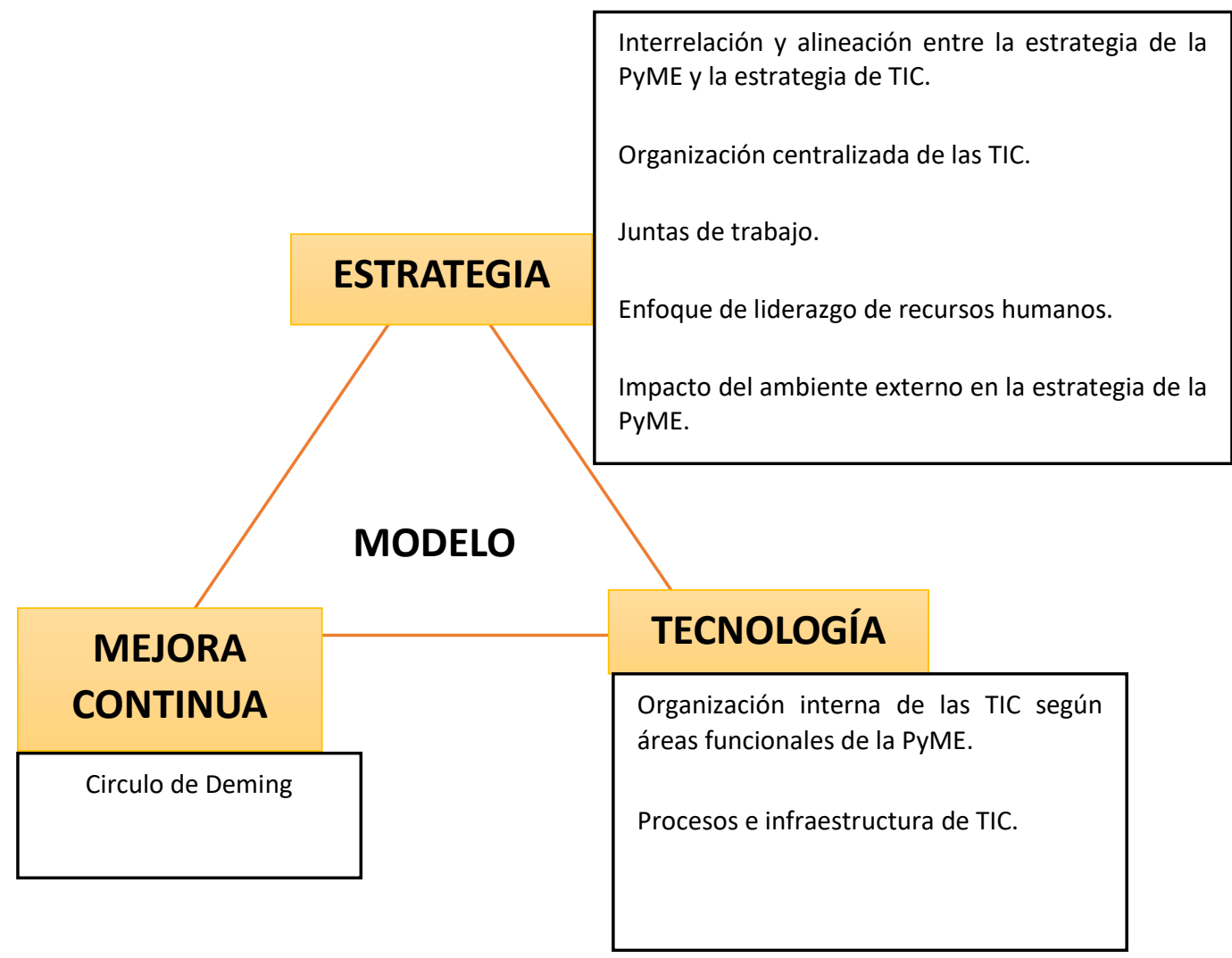

Fuente: elaboración propia. 


\section{VARIABLES DEL MODELO}

La operacionalización de las variables se relaciona con el grado de abstracción de éstas y es el procedimiento que tiende a pasar de las variables generales a las intermedias (dimensiones) y de éstas a los indicadores. En la tabla 1 se presentan las nueve variables del modelo con sus subvariables.

Tabla 1. Variables y subvariables del Modelo de Gestion de las TIC.

\begin{tabular}{|c|c|}
\hline Variable del modelo & Componentes / subvariables \\
\hline \multirow{2}{*}{ Estrategia de la PyME } & Alcance de la empresa \\
\hline & Gobierno corporativo \\
\hline \multirow{3}{*}{$\begin{array}{l}\text { Procesos e infraestructura } \\
\text { organizacional }\end{array}$} & Estructura administrativa \\
\hline & Volúmenes y valores de producción y de ventas \\
\hline & Competencias de recursos humanos \\
\hline \multirow{4}{*}{ Estrategia de las TIC } & Aplicaciones básicas \\
\hline & Competencias sistémicas \\
\hline & Gobierno de las TIC \\
\hline & Riesgos de las TIC \\
\hline \multirow{2}{*}{ Procesos e infraestructura de TIC } & Infraestructura \\
\hline & Procesos \\
\hline Juntas de trabajo & Frecuencia de las reuniones \\
\hline \multirow{4}{*}{ Circulo de Deming } & Diagnóstico, planificación y ajuste estratégico \\
\hline & Ejecución de lo planificado \\
\hline & Evaluación \\
\hline & Ajuste \\
\hline $\begin{array}{c}\text { Organización de las TIC a } \\
\text { Nivel corporativo }\end{array}$ & Tipo de organización de las TIC a nivel corporativo \\
\hline $\begin{array}{l}\text { Organización de las TIC en el plano } \\
\text { interno }\end{array}$ & Tipo de organización de las TIC en el plano interno \\
\hline \multirow{2}{*}{$\begin{array}{l}\text { Alineación de las TIC con la } \\
\text { estrategia de la empresa }\end{array}$} & Financiera \\
\hline & Estratégica \\
\hline
\end{tabular}

\section{VALIDACIÓN DE LA PROPUESTA MEDIANTE LA CONSULTA DE EXPERTOS}

A los efectos de la validación del Modelo de Gestión de las TIC para las PyMEs de Riobamba, se realiza una consulta a los expertos, para lo cual se diseña un documento para solicitar sus opiniones.

El método de criterio de expertos en la investigación, es un método cualitativo de pronóstico y comprobación, usado desde la década de 1950 (González, s.f.).

Para el objetivo de esta investigación como expertos en TIC se escogió académicos en el área de informática y expertos en el área de las PyMEs con la experiencia en la dirección de las empresas. En ambos casos se tomaron en cuenta: los títulos académicos, años de experiencia (más de 15 años), cargos desempeñados, publicaciones científicas, participación en los 
proyectos de investigación y en la elaboración de materiales metodológicos, proyectos estratégicos.

Durante los primeros contactos con los posibles expertos se les informa acerca de los objetivos del estudio, criterios de selección, usos potenciales del Modelo.

A los potenciales expertos se aplicó un cuestionario de autovaloración, para determinar su nivel de competencia. Las respuestas obtenidas se compararon con la tabla de valores establecidos para 6 items.

Para determinar el nivel de competencia (Hurtado, s.f.) se utiliza el Coeficiente de Competencia (K) se obtiene sumando los coeficientes de conocimiento (Kc) y de argumentación (Ka). En el Anexo 1 se presenta el Cuestionario de Autovaloración conjuntamente con la tabla de valores preestablecidos.

Para que el experto se pueda considerar como competente debe obtener $6>=\mathrm{K}>=4,5$

El número de expertos no es determinado y depende entre otras cosas de existencia de los mismos y de voluntad de ellos de participar en esta parte de investigación.

En este caso el cuestionario se envió a los 15 potenciales expertos y a partir de esta autoevaluación, basándose en las respuestas obtenidas, se seleccionaron 12 expertos para valoración de la propuesta los mismos que obtuvieron Coeficiente de Competencia mayor a 4,5 .

Con los expertos seleccionados se conforma el panel, o sea el conjunto de expertos que tomarán parte en el Delphi. El método Delphi (Scott, 2001; Luna, 2005) es una técnica basada en un panel de expertos, de comunicación estructurada, que sirve para obtener información esencialmente cualitativa.

Entre las características de este método destacan (Varela, 2012): no se requiere llegar a un consenso, es utilizable entre grupos geográficamente dispersos y es anónimo, puesto que los expertos no se conocen entre sí.

Los instrumentos utilizados en el método Delphi son: formularios, correo electrónico, video conferencia, comunicación directa.

Este método consiste en solicitar en forma sistemática las opiniones de un grupo de expertos.

Para tal efecto se les hace llegar el resumen de la propuesta conjuntamente con el instrumento de evaluación, con el fin de que evalúen la misma otorgando el valor correspondiente a cada uno de los seis criterios establecidos.

Durante el proceso de aplicación del instrumento se realizan algunos intercambios de opiniones entre la investigadora y los expertos. Estos se consideran para modificar algunos aspectos relacionados con el Modelo y así mejorar la propuesta del Modelo de Gestión de las TIC en las PyMEs. En la tabla 2, se muestran los valores de los rangos de las calificaciones emitidas por los expertos para cada uno de los seis criterios de evaluación ponderados de 1 a 5 , correspondiendo el 5 a la máxima calificación. 


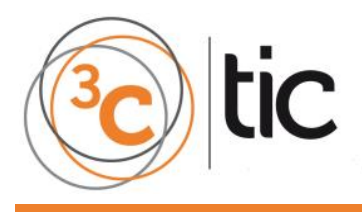

3C TIC (Edición 23) Vol.6 - №4

Diciembre'17-marzo'18, 1 - 18

Área de Innovación y Desarrollo, S.L.

ISSN: 2254-6529

DOI: http://dx.doi.org/10.17993/3ctic.2017.58.1-18

Tabla 2. Resultados del procesamiento de las opiniones de los expertos sobre la validez de la propuesta presentada.

\begin{tabular}{|c|c|c|c|c|c|c|c|}
\hline Expertos & $\begin{array}{c}\text { Pertinencia } \\
y \\
\text { actualidad de } \\
\text { la propuesta }\end{array}$ & $\begin{array}{l}\text { Consisten } \\
\text { cia teórica }\end{array}$ & $\begin{array}{c}\text { Cohere } \\
\text { ncia } \\
\text { lógica }\end{array}$ & $\begin{array}{c}\text { Factibilidad } \\
\text { de la } \\
\text { propuesta }\end{array}$ & $\begin{array}{l}\text { Importan } \\
\text { cia e } \\
\text { impacto }\end{array}$ & $\begin{array}{c}\text { Contribución } \\
\text { a la mejora } \\
\text { organizaciona } \\
\text { I }\end{array}$ & $\Sigma$ \\
\hline E1 & 5 & 4 & 4 & 5 & 5 & 5 & 28 \\
\hline E2 & 5 & 5 & 4 & 5 & 5 & 4 & 28 \\
\hline E3 & 5 & 4 & 5 & 4 & 5 & 5 & 27 \\
\hline E4 & 4 & 5 & 5 & 5 & 5 & 4 & 28 \\
\hline E5 & 5 & 5 & 5 & 5 & 5 & 4 & 29 \\
\hline E6 & 4 & 4 & 5 & 5 & 5 & 5 & 28 \\
\hline E7 & 5 & 5 & 5 & 4 & 4 & 5 & 28 \\
\hline E8 & 5 & 4 & 5 & 4 & 5 & 5 & 27 \\
\hline E9 & 4 & 5 & 4 & 5 & 5 & 5 & 28 \\
\hline E10 & 4 & 4 & 5 & 4 & 5 & 5 & 26 \\
\hline E11 & 5 & 3 & 5 & 4 & 5 & 5 & 28 \\
\hline E12 & 5 & 5 & 4 & 5 & 5 & 5 & 27 \\
\hline$\Sigma$ & 56 & 55 & 56 & 55 & 59 & 57 & 338 \\
\hline Peso relativo & $56 / 338$ & $55 / 338$ & $56 / 338$ & $55 / 338$ & $59 / 338$ & $57 / 338$ & \\
\hline Ponderación & 0,165 & 0,163 & 0,165 & 0,163 & 0,175 & 0,169 & 1,0 \\
\hline \multirow[t]{2}{*}{$\begin{array}{c}\text { Media } \\
\text { aritmética }\end{array}$} & 4,67 & 4,58 & 4,67 & 4,58 & 4,92 & 4,75 & 28,17 \\
\hline & & & & & & $\begin{array}{c}\text { Promedio } \\
\text { general }\end{array}$ & 4,7 \\
\hline
\end{tabular}

Fuente: elaboración propia.

Las calificaciones otorgadas por los expertos a cada uno de los criterios se encuentran entre los valores 3 y 5 . Las medias se hallan entre 4,58 y 4,92; siendo el promedio general 4,7. Por esta vía se ratifica la validez del contenido de la propuesta.

Por otra parte, los rangos de las calificaciones conferidas por los especialistas para cada criterio se someten a una prueba de fiabilidad, que consiste en determinar la coincidencia entre los expertos; para ello, se aplica el método ANOCHI (Moreno y Rodríguez, 2013;Madrid, 2013). En este método, primeramente se calcula la discrepancia del rango.

Según la Tabla para Determinar la Diferencia de Rango Máximo en la evaluación de n jueces, la discrepancia máxima para 12 expertos y un rango de 1-7, es de 216 . Dividiendo la discrepancia del criterio entre la discrepancia máxima, se obtiene el índice de discrepancia. Al considerar su complemento queda determinado el índice de coincidencia. Los valores obtenidos, según establece el método se muestran en la tabla 3. 
Tabla 3. Resultados del método ANOCHI aplicado a las valoraciones de los expertos sobre el modelo.

\begin{tabular}{|c|c|c|c|c|c|c|c|}
\hline $\begin{array}{c}\text { Índices } \\
\text { derivados }\end{array}$ & $\begin{array}{c}\text { Pertinencia } \\
\mathbf{y} \\
\text { actualidad } \\
\text { de la } \\
\text { propuesta }\end{array}$ & $\begin{array}{c}\text { Consist } \\
\text { encia } \\
\text { teórica }\end{array}$ & $\begin{array}{l}\text { Coheren } \\
\text { cia lógica }\end{array}$ & $\begin{array}{l}\text { Factibilida } \\
\text { d de la } \\
\text { propuesta }\end{array}$ & $\begin{array}{c}\text { Importancia } \\
\text { e impacto }\end{array}$ & $\begin{array}{c}\text { Contribució } \\
\text { n a la } \\
\text { mejora } \\
\text { organizacion } \\
\text { al }\end{array}$ & $\begin{array}{l}\text { Medi } \\
\quad \text { a }\end{array}$ \\
\hline $\begin{array}{c}\text { Discrepancia } \\
\text { de rango }\end{array}$ & 32 & 46 & 32 & 35 & 11 & 27 & 30,5 \\
\hline $\begin{array}{c}\text { Índice de } \\
\text { discrepancia }\end{array}$ & $\begin{array}{c}32 / 216= \\
0,148\end{array}$ & 0,21 & 0,148 & 0,16 & 0,05 & 0,12 & 0,14 \\
\hline $\begin{array}{c}\text { Índice de } \\
\text { coincidencia }\end{array}$ & $\begin{array}{c}1-0,148= \\
0,85\end{array}$ & 0,79 & 0,85 & 0,84 & 0,95 & 0,88 & 0,86 \\
\hline
\end{tabular}

Fuente: elaboración propia.

El método ANOCHI establece una fiabilidad aceptable o buena fiabilidad para un índice de coincidencia entre el rango 0,61- 0,80 y elevada o buena cuando es superior a 0,80 (Mayagoitia, 2016). El índice de coincidencia obtenido es de 0,86 , por lo cual queda comprobada la validez de la propuesta por los resultados obtenidos mediante la consulta a los expertos.

También se entrega la propuesta a la Cámara de Comercio de Riobamba para su respectiva evaluación, obteniendo una valoración muy positiva.

\section{VISIBILIDAD DE LA PROPUESTA}

\subsection{VIABILIDAD ADMINISTRATIVA - ORGANIZACIONAL}

Existe la viabilidad administrativa - organizacional para la ejecución de la propuesta como lo confirma el siguiente análisis:

- La propuesta parte de estudios de diagnóstico de utilización de las TIC en las PyMEs de Riobamba, donde se evidencia la falta de aplicación plena de estas herramientas. En consecuencia el Modelo de Gestión de las TIC está debidamente sustentado en las necesidades y realidades de este tipo de empresa.

- Los gerentes de las PyMEs riobambeñas son conscientes de los beneficios que brindan las TIC para las empresas, por lo cual estarán abiertos a la posibilidad de adaptar un Modelo para mejorar la gestión de estas herramientas.

- Actualmente gracias a las nuevas tecnologías existe mucho más apoyo para la creación de nuevas empresas. Crear una empresa brinda oportunidades a los emprendedores para que desarrollen sus capacidades y aporten positivamente al sistema económico del país. Sin embargo, para que una empresa sea conocida y competitiva, es necesario gestionar las TIC eficientemente para que aporten al logro de los objetivos del negocio.

- En el nuevo contexto económico abierto, las PyMEs se ven obligadas a mejorar constantemente sus recursos tecnológicos y humanos y a realizar alianzas estratégicas con otras empresas e instituciones. 


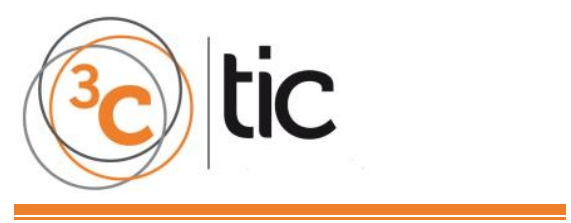

- La filosofía de gestión expresada en la misión, visión y principios de muchas PyMEs, contiene en sus enunciados la importancia que se da al efectivo manejo de los recursos tecnológicos.

- El estado ecuatoriano apoya e incentiva la utilización de estas herramientas tecnológicas.

- Hay espacio para la internacionalización de bienes y servicios con el uso de las TIC.

- Tanto en el ámbito privado como en público, existe la posibilidad de realizar muchos trámites con estas herramientas.

\subsection{VIABILIDAD TÉCNICA / TECNOLÓGICA}

La viabilidad técnica / tecnológica es muy importante en este trabajo, se basa en las siguientes realidades:

- En Riobamba, como en la mayor parte de Ecuador, existe una sólida infraestructura de conectividad y telecomunicaciones.

- Existe disponibilidad de la tecnología avanzada en el Ecuador.

- En Riobamba hay disponibilidad de recursos humanos capacitados en área de sistemas, pues cada año se gradúan en la Escuela Superior Politécnica de Chimborazo profesionales de esta área.

- Existe infraestructura de las TIC disponible al interior de las empresas, como lo muestran los resultados de las encuestas aplicadas (Slusarczyk, 2015a), más del 95\% de las PyMEs cuentan con una base para la aplicación del Modelo que son: computadoras, software básico e Internet, los cuales se constituyen en herramientas básicas.

- En Ecuador existen algunas empresas desarrolladoras de software especializado para las PyMEs

- Muchos profesionales del área de sistemas están migrando a Quito en busca de trabajo, por lo cual sería importante brindar oportunidad para ellos como jefes de las TIC en las empresas de Riobamba.

\subsection{VIABILIDAD ECONÓMICA}

La viabilidad económica se respalda en los siguientes hechos:

- La propuesta se llevará a cabo dentro de las instalaciones de las PyMEs, lo que permitirá la utilización de recursos infraestructurales, técnicos y humanos existentes para proceder con la implantación del Modelo.

- La inclusión en la propuesta de las Juntas de trabajo no conlleva costos adicionales, solamente exige decisión y un mejor liderazgo.

- Existen soluciones informáticas gratuitas que pueden ser instaladas para mejorar el desempeño de las empresas como por ejemplo: Skype para videoconferencias, Gimp: para editar imágenes, Google Sketchup para crear dibujos en 3D etc., programa gratuito para la gestión y administración de PyMEs por ej. SmartNetAdmin.

- La empresa puede optar por la computación en la nube (Cloud computing). Este tipo de solución evita a la empresa realizar fuertes inversiones en tecnología en un solo momento y además permite planificar los gastos, pues el pago por ese tipo de servicio es constante. Hay soluciones generales que abarcan desde la gestión de relaciones con clientes (CRM) a la facturación, la colaboración y el apoyo. Algunas de estas soluciones son: WORKetc, Zoho, BlueCamroo, Sellsy, Bitrix, 1CRM, ExpertApps. 


\section{CONCLUSIONES}

- El Modelo de Gestión de las TIC en las PyMEs presentado integra los elementos del entorno, concibe herramientas para la alineación entre las TIC y las estrategias de la organización y sienta las pautas para la colaboración entre los diferentes responsables de la toma de decisiones en las PyMEs.

- La aplicación del Modelo aporta una mejor gestión, control, y toma de decisiones, lo que puede elevar el desarrollo productivo y socioeconómico de estas empresas, por lo cual está en concordancia con las políticas del gobierno ecuatoriano, que pretende reforzar la matriz productiva del país.

- Los expertos que evaluaron y validaron la propuesta presentada, reconocen su importancia, pertinencia, actualidad, consistencia teórica, coherencia lógica, factibilidad en el contexto riobambeño y contribución a la mejora organizacional, con lo cual se demuestra la pertinencia del Modelo.

- Existe la viabilidad administrativa - organizacional, técnica - tecnológica y económica para la implementación del Modelo para la Gestión de las TIC en las PyMEs de Riobamba. 


\section{REFERENCIAS BIBLIOGRÁFICAS}

1. Araque, W. (2015). Caracterización de la Pyme ecuatoriana. Gestión, 64-67. Ecuador.

2. Barrera, M. (2010). Las pequeñas y medianas empresas ecuatorianas. Quito- Ecuador: Cámara de la Pequeña Industria de Pichincha.

3. Barroso, M. (2005). Meditaciones Gerenciales. Editorial Galac.

4. Constante, L. y Quintana, W. (2014). Alineamiento de las Tecnologías de la Información y Comunicaciones (TIC's) con la estrategia del negocio en las PYMES. Quito. Tesis Maestría en Gestión de las Comunicaciones y Tecnologías de la Información (FIS). Obtenido de: http://bibdigital.epn.edu.ec/handle/15110/8993

5. Deming, E. (2008). Calidad, productividad y competitividad (3a). Madrid-España: Díaz de Santos.

6. García, E. (2016). El Ciclo de Deming: La gestión y mejora de procesos. Obtenido de: http://equipo.altran.es/el-ciclo-de-deming-la-gestion-y-mejora-de-procesos/

7. Gimbert, X. (2007). El enfoque estratégico de la empresa. DEUSTO S.A. EDICIONES

8. Global CIO Study 2009, IBM. Obtenido de: http://www05.ibm.com/services/es/cio/pdf/CIE03046-ESES-00 LDG CHG.pdf

9. González, C. (Septiembre 2012). Enfoque tecnológico de la organización. Obtenido de: https://prezi.com/nattapvy mt2/enfoque-tecnologico-de-la-organizacion/

10. González, M.V. (s.f.). Valoración por el método del criterio de expertos. Obtenido de: http://www.eumed.net/librosgratis/2009d/620/Valoracion/por/elmetodo/ delcriteriode/expertos.htm

11. Hidalgo M., Proaño, C., Sandoval M. (2011). Evaluación del uso de las TICs en el desempeño de las PYMES ubicadas en la zona urbana de la ciudad de Latacunga. Tesis de grado ESPE. Ecuador.

12. Hurtado, S. (s.f.) Criterio de expertos. Su procesamiento a través del Método Delphy. Obtenido de: http://www.ub.edu/histodidactica/index.php?option=com content\&view=article\&id =21:criterio-de-expertos-su-procesamiento-a-traves-del-metodo-

delphy\&catid=11: metodologia-y-epistemologia\&ltemid $=103$

13. IT Governance Institute. (2007). COBIT 4.1: Framework, Control Objectives, Management Guidelines, Maturity Models.

14. Kast, F. E. (1992). Administración en las organizaciones: enfoque de sistemas y de contingencias. México: McGraw Hill.

15. Kearney, A.T. (2005). Informe sobre globalización. Disponible en: http://www.atkearney.com/shared res/pdf/2005G-index.pdf

16. Luftman, J. (2000). Assessing Business - IT alignment maturity. Communications of AIS, 4. Article 14. 2000.

17. Luna, H.P. y otros. (2005). Los Delphi como fundamento metodológico predictivo para la investigación en sistemas de información y tecnologías de la información (IS/IT). Pixel-Bit. Revista de Medios y Educación, (26): 89-112, 2005, ISSN: 1133-8482.

18. Madrid, A. y otros. (2013). Diseño de una metodología para la implementación de un repositorio electrónico de conocimiento. Revista Internacional Administración \& Finanzas, 6(5). Obtenido de: http://www.theibfr2.com/RePEc/ibf/riafin/riaf-v6n52013/RIAF-V6N5-2013-1.pdf

19. Malaret, J. (2003). Liderazgo de equipos con entusiasmo estratégico. España: Ed. Díaz de Santos.

20. Mayagoitia, E. y otros. (2016).Vulnerabilidad escolar en educación media superior. Obtenido de: http://altamira.cut.edu.mx/assets/archivos/articulos/58e30fd13c18e5.VULNERABILIDAD\%20ESCOLAR\%20EN\%20EDUCACIO\%CC\%81N\%20MEDIA\%20SUPE RIOR.pdf 


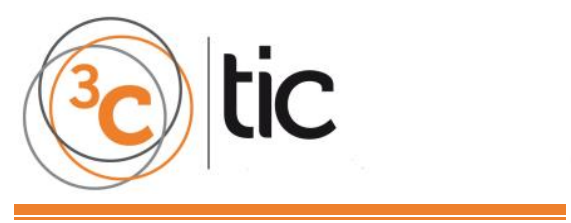

21. Ministerio de Relaciones Laborales. (2015). Datos de la Generación de Empleo de las PYMES en la ciudad de Riobamba. Riobamba.

22. Ministerio de Telecomunicaciones y de la Sociedad de la Información. (2014). Tecnologías de Información y Comunicación para el desarrollo. Reporte, Subsecretaria de Fomento a la Sociedad de la Información y Gobierno en Línea, Quito: MINTEL.

23. Molina, D. (2014). Nuevo modelo de gobernanza de las TIC. boletic 71. Obtenido de: http://www.astic.es/sites/default/files/articulosboletic/mono6 doming molina.pdf

24. Monge, R. (2005). TIC en Las PYMES de Centroamérica: Impacto de la Adopción de Las Tecnologías de la Información Y la Comunicación en El Desempeño de Las Empresas. Costa Rica, IDRC.

25. Moreno, N. y Rodríguez F. (2013). Método para determinar la concordancia entre expertos como medida de fiabilidad. Ponencia presentada en el Congreso Internacional de Investigación Científica Multidisciplinaria (ICM13), ITESM, Chihuahua, 2013.

26. Ochoa, M. y otros. (2007). Innovación, tecnología y gestión tecnológica. Acimed, 16(4).

27. Peirano, F. y Suárez, D. (2006). TICS y empresas: propuestas conceptuales para la generación de indicadores para la sociedad de la información. Journal of Information Systems and Tecnology Managment, 3(2),2006,p.123-142.

28. Ramón, J. (14 de 06 de 2014). ¿Cuánto deben gastar las empresas en informática? Obtenido de: http://informatica.blogs.uoc.edu/2014/06/16/cuanto-deben-gastarlas-empresas-en-informatica/

29. Romero, J. (2011). El impacto de las TICs en el desempeño de las PYMES en Ecuador, cantón Riobamba, provincia de Chimborazo, año 2010. Tesis de grado UTPL. Ecuador.

30. Sánchez, V. G. (2011). Administración de Empresas. Ediciones Pirámide.

31. Scott, G (2001). Strategic Planning for High-Tech Product Development. Technology Analysis \& Strategic Management, 13(3).

32. Slusarczyk, M. (2015a). Diagnóstico de la aplicación de las NTIC en las PYMES de Riobamba -Ecuador. 3C TIC, cuadernos de desarrollo aplicados a las TIC, 4(2), 145168. Junio 2015.

33. Slusarczyk, M. (2015b). Análisis de estrategias y modelos de aplicación de las TIC en las empresas. Revista Científica ECOCIENCIA, 6(2). Diciembre 2015.

34. Torres, C. (2015). Modelo para la gestión de políticas territoriales de desarrollo local a escala municipal en Cuba. Tesis presentada en opción al grado científico de Doctor en Ciencias Económicas. Universidad de La Habana.

35. Universidad de Champagnat. (2002). Administración proactiva para la previsión y solución de problemas. Obtenido de: https://www.gestiopolis.com/administracionproactiva-para-la-prevision-y-solucion-de-problemas/

36. Varela, M. y otros. (2012). Descripción y usos del método Delphi en investigaciones del área de la salud. Investigación en Educación Médica,1(2), 90-95. Obtenido de: http://riem.facmed.unam.mx/sites/all/archivos/V1Num02/07 MI DESCRIPCION Y USOS.PDF

37. Winkler, T. (2014). Information Management and IT Strategy. Material de lectura correspondiente a la Escuela de Verano Internacional en Economía y Gestión. La Habana. 